\title{
User-Centric Spectrum Sharing in Dynamic Network Architecture
}

\author{
Alireza Shams Shafigh and Savo Glisic \\ Centre for Wireless Communications, University of Oulu \\ P.O. Box 4500, 90014 University of Oulu, Finland \\ Email: \{ashamssh, savo.glisic\}@ee.oulu.fi
}

\begin{abstract}
Dynamic network architecture (DNA) has emerged as a promising networking paradigm which establishes a low-cost ubiquitous network connectivity without the need for significant changes in software and hardware infrastructure. In this concept the major player in spectrum sharing process is the user itself hence the name user-centric spectrum sharing. Such user-based spectrum sharing concept is an opportunistic model due to the uncertainty in traffic patterns, which results in frequent interruption of the shared spectrum access. In this paper, end users (EUs) deal with this difficulty by choosing the least overloaded access points (APs) to have a longer access period without interruption. Moreover, we introduce spectrum outage probability parameter to investigate efficiency of spectrum sharing in DNA networks. The simulation results show that the network operators (NOs), dynamic access points (DAPs) and EUs significantly improve their utilities if they deploy a user-centric spectrum sharing model in DNA networks.
\end{abstract}

Keywords. Dynamic network architecture, spectrum sharing, Stackelberg game, network utility maximization.

\section{INTRODUCTION}

Dynamic network architecture (DNA)/user provided network (UPN) paradigm [1], [2], [3] introduces a low-cost ubiquitous Internet connectivity for both in coverage and out of coverage users without need for additional infrastructure. For this reason, UPN/DNA has been recently introduced by 3GPP as a complementary communication and network scheme [4]. DNA enables users of mobile virtual network operators (MVNOs) to share their unused resources e.g. bandwidth with other users [1], [3] especially once users are out of coverage. End user (EU) becomes a resource provider by acting as access point (AP)/relay and sharing temporally unused resources according to the offered incentives by NOs [5], [6].

In [7], a new business model is provided for UPN/DNA model which is more efficient than the old ones, but it is not suitable for ultra-dense wireless networks with multiple operators. In [8], interactions between operator and mobile users is formulated as a two-stage game. The literature shows that mobile users memberships (EU or AP) can significantly influence the performance of the network. Crowdsourced Wireless Community Networks [9], [10], and [11] enable mobile users to connect with each other and share their Internet connection.

In [12], authors consider a multiple operators scenario where macro-cell base stations compete to gain access to femto-cell access points in order to reduce their traffic loads. In [13], for the same scenario, a solution has been proposed which jointly associates the user equipment (UE) to the APs, and allocates the Femto APs to the service providers (SP) in such a way that the total satisfaction of the UEs in an uplink OFDMA network is maximized.

In [3], we proposed DNA as a new networking model, where network operators can motivate their users to act as access points. In this paper, DNA as a new dynamic spectrum sharing model is proposed to improve the flexibility, the adaptability, and the efficiency of wireless communication systems for both in coverage and out of coverage EUs. In DNA, the unused frequency spectrum/bandwidth can be efficiently shared among licensed or even unlicensed EUs. This model has a hierarchical structure in which the network operators (NOs) share their spectrum/bandwidth with DNA access points (DAPs), and the DAPs opportunistically share their unused spectrum/bandwidth with EUs. In order to show the proposed user-based sharing model is suitable for the ultra-dense network, we use a new performance metric called interruption probability which indicates how long the shared spectrum can be used by EUs without interruption. We elaborate this parameter in order to clarify how the spectrum sharing can be successful if DAPs has no prior knowledge about their traffic.

The rest of the paper is organized as follows. Section II provides the general system model. Sections III explains the DNA-based spectrum sharing concept. Section IV provides the numerical results and finally, Section V concludes the paper.

\section{General System Model}

We consider a DNA network [3] assuming $N$ wireless network operators using $J$ static/dynamic access points to serve $K$ end users, where $\mathcal{N}, \mathcal{J}$ and $\mathcal{K}$ are the lists of NOs (Network Operators), APs (Access points) and EUs (End Users) respectively. Each NO serves two different types of users depending on its coverage range, normal users (NUs) and end users (EUs) which are in and out of coverage respectively. NUs are served directly by their corresponding NOs while out of coverage EUs might be served by APs provided by DNA networking paradigm.

We assume that every NO $n$ owns $W_{n}$ as total bandwidth for providing proper service for its $I_{n}$ Normal Users (NUs). The unused portion of the spectrum can be shared with DAP. NO $n$ can be specified by tuple $\left(W_{n}, I_{n}, A_{n}, \beta_{n}, P_{n}, p_{n}\right)$, where it shares $A_{n}$ out of $W_{n}$ bandwidth and $\beta_{n}$ represents an incentive 
rate for DAPs. The shared spectrum $A_{n}=\sum_{j \in \mathcal{J}} A_{n, j}$ is the sum of allocated spectrum to DAPs. $p_{n}$ and $P_{n}$ represent the prices of the spectrum for EUs and DAPs respectively. NO $n$ serves its NUs using the unshared spectrum $W_{n}-\sum_{j \in \mathcal{J}} A_{n, j}$ out of $W_{n}\left(\frac{W_{n}-\sum_{j \in \mathcal{J}} A_{n, j}}{I_{n}}\right.$ for each NU) and shares the remaining spectrum $\sum_{j \in \mathcal{J}}^{I_{m}} A_{n, j}$ with DAPs, where $A_{n, j}$ is the shared spectrum by NO $n$ with DAP $j$. In the same way, every DAP $j$ shares its possible unused spectrum $\sum_{k \in \mathcal{K}} a_{j, k}$ with EUs which belong to different NOs, where $a_{j, k}$ is the shared spectrum by DAP $j$ with EU $k$. The set of EUs accessing the spectrum of the same DAP $j$ creates cluster $\mathcal{L}_{j}$. Members of each cluster use spectrum of the corresponding DAP known as cluster head. Let us denote the set of EUs using with EU $k$ in access to the shared spectrum of DAP $j$ as $\mathcal{L}_{j}(k)$ (See Fig.1). In the proposed model, every EU can get spectrum from different DAPs.

Overall, there are two different markets in the proposed model in Fig.1, where the first market is between NOs as suppliers and DAPs as originators of demands and the second market consists of DAPs as spectrum suppliers and EUs as originators of demands. In this paper, we use market equilibrium point as solution of this spectrum sharing markets. In the equilibrium point demands and supplies are equal. Fig.1 presents a hybrid spectrum sharing in DNA network, where every NO $n$ shares $A_{n}$ out of $W_{n}$ bandwidth and $\left(W_{n}-A_{n}\right)$ is distributed among NUs. NO $n$ assigns $A_{n, j}$ to every DAP $j$ demanding $D_{j, n}$ at the price $P_{n}$. Parameters $A_{n, j}$ and $D_{j, n}$ are equal in the market equilibrium point. Then, every DAP $j$ shares $a_{j}$ out of $\min \left(A_{n, j}, D_{j, n}\right)$ with EUs. EUs demand all together $d=\sum_{k \in \mathcal{K}} \sum_{j \in \mathcal{J}} d_{k, j}$ bandwidth from DAPs, where $d_{k, j}$ is the demand of EU $k$ from DAP $j$. In the second market's equilibrium point, $a_{j, k}$ and $d_{k, j}$ are equal. With all this in mind, the sharing rate of every DAP $j$ is given by

$$
\alpha_{j}=\frac{\sum_{k \in \mathcal{K}} \min \left(a_{j, k}, d_{k, j}\right)}{\sum_{n \in \mathcal{N}} \min \left(A_{n, j}, D_{j, n}\right)} .
$$

In the above, $\min \left(A_{n, j}, D_{j, n}\right)$ and $\min \left(a_{j, k}, d_{k, j}\right)$ ensure that suppliers (NOs and DAPs) can not share more than their available spectrum and consumers (DAPs and EUs) do not get more spectrum than their demands. According to (1), every DAP $j$ assigns $\alpha_{j} \min \left(A_{n, j}, D_{j, n}\right)$ to EUs and $\left(1-\alpha_{j}\right) \min \left(A_{n, j}, D_{j, n}\right)$ to itself.

In the proposed spectrum sharing model, DAPs use their available spectrum for own usage and share the unused spectrum with EUs. Considering unknown traffic patterns of DAPs, they have to reclaim their shared spectrum. This may make some interruption to EUs' communications. In the user-centric spectrum sharing mechanism, interruption probability is the main parameter which reflects both benefit and complexity of the DNA networking model.

Definition 1. Interruption rate is the probability that a DAP recaptures its spectrum which already has been assigned to an $E U$.

The interruption rate $\gamma_{j}$ depends on the traffic variation of DAP $j$. The interruption rate quantifies degradations of the performance of the spectrum sharing mechanism in the second market. Thus, it should be included as a penalty term in utility

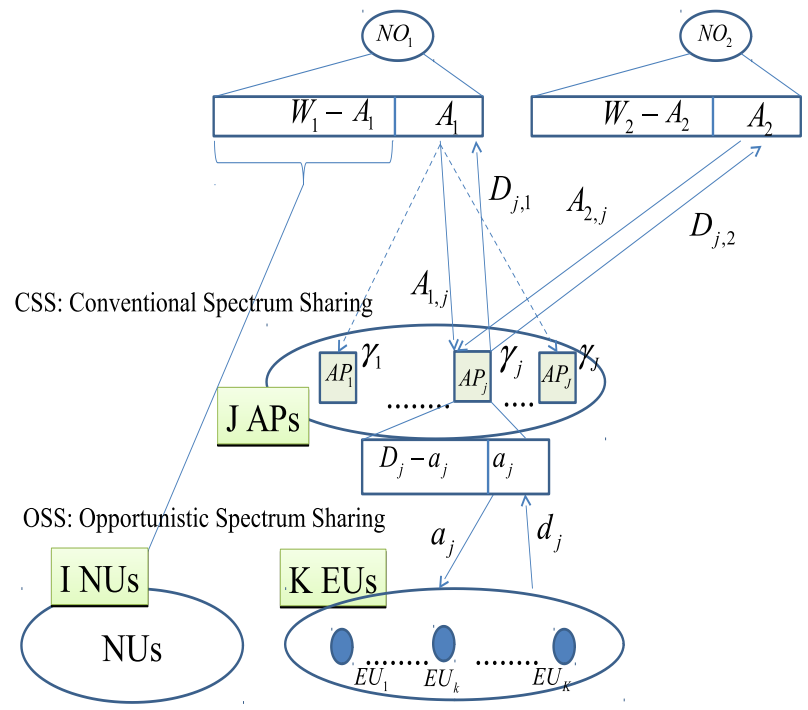

Fig. 1. System model.

function to force DAPs to provide more reliable access for EUs.

We assume that every DAP $j$ uses time division multiple access (TDMA) as its channel access method to share its unused bandwidth with EUs. TDMA allows several EUs to share the same frequency channel of bandwidth $\sum_{n \in \mathcal{N}} \min \left(A_{n, j}, D_{j, n}\right)$ in different time slots. The average interruption probability of an ongoing EU's transmission in a given time slot can be calculated by

$$
\gamma_{j} \cong \sum_{z=1}^{\infty} \frac{\kappa_{j}^{z} \exp \left(-\kappa_{j}\right)}{z !},
$$

where $\frac{\kappa_{j}^{z} \exp \left(-\kappa_{j}\right)}{z !}$ is the probability of having $z \geq 1$ packet arrivals following a Poisson distribution. Parameter $\kappa_{j}$ represents arrival rate. The probability that an allocated channel is used by EUs without interrupting by DAP $j$ is $1-\gamma_{j}=\exp \left(-\kappa_{j}\right)$ $(z=0$ in (2)). The average shared spectrum by every DAP can be calculated by $\frac{\sum_{j \in \mathcal{J}} a_{j} e^{\left(-\kappa_{j}\right)}}{J}$. Here, we should investigate spectrum outage probability of EUs when none of DAPs can provide spectrum during a period of time.

Definition 2. The spectrum outage probability (SOP) is the probability that none of DAPs are able to provide at least one free time slot during $\delta$ consecutive time slots, where $\delta$ is the number of time slots. We can calculate SOP for a DNA network by

$$
O=\prod_{j \in \mathcal{J}} \gamma_{j}^{\delta}=\prod_{j \in \mathcal{J}}\left(1-\exp \left(-\kappa_{j}\right)\right)^{\delta},
$$

\section{DNA-BASEd Spectrum Sharing Scheme}

A simplified single-operator scenario is investigated to clarify the DNA spectrum sharing model in more detail. So, we drop $n$ from formulations. Notice that the notations $X_{J \times N}$ and $Y_{J \times K}$ are used throughout the paper to denote the equilibrium points of the first and second markets, where $X_{j, n}$ and $Y_{j, k}$ $(j \in \mathcal{J}, n \in \mathcal{N}, k \in \mathcal{K})$ represent demands of DAP $j$ and EU $k$ from NO $n$ and DAP $j$ respectively. Also $X_{j, n}$ and $Y_{j, k}$ 
represent the shared spectrum (equal with demands) by NO $n$ and DAP $j$ with DAP $j$ and EU $k$ respectively.

\section{A. Closed-Form Formulation}

In a single-operator scneario, NO shares the spectrum $A$ out of $W$ with APs and the remaining spectrum $W-A$ is distributed between its NUs. The utility function for every NU can be calculated by $\ln \left(\frac{q(W-A)}{I}\right)$, where $q$ is the channel efficiency of spectrum for NUs. Every DAP $j$ receives spectrum $\frac{A \alpha_{j}}{\sum_{j^{\prime} \in \mathcal{J}} \alpha_{j^{\prime}}}$ out of total shared spectrum $A$ proportional to its relative sharing rate $\frac{\alpha_{j}}{\sum_{j^{\prime} \in \mathcal{J}^{\alpha}} \alpha_{j^{\prime}}}$. This means that NO distributes the spectrum $A$ proportional to the sharing rates of APs to encourage them to cooperate by increasing their sharing rates. We use notations $D$ and $d$ to denote the demands of DAPs and EUs from NO and DAPs respectively. Furthermore, we assume that all DAPs have similar traffic distribution and hence equal interruption rate $\gamma$. As in the equilibrium points of both markets, demands and supplies are equal, let $X=\sum_{j \in \mathcal{J}} X_{j}=\sum_{j \in \mathcal{J}} \min \left(A_{j}, D_{j}\right)$ and $Y=\sum_{j \in \mathcal{J}} Y_{j}=$ $\sum_{j \in \mathcal{J}} \sum_{k \in \mathcal{K}} \min \left(a_{j, k}, d_{k, j}\right)$ be the equilibrium points of the first and second markets. In the first market, $A_{j}$ and $D_{j}$ are the shared spectrum by NO for DAP $j$ and demand of DAP $j$ from NO respectively. Likewise, in the second market, $a_{j, k}$ is the shared spectrum by DAP $j$ for EU $k$ and $d_{k, j}$ is demand of EU $k$ from DAP $j$. Profit function of NO can be presented as

$$
\begin{aligned}
& \pi=I \ln \left(\frac{q(W-X)}{I}\right)+ \\
& P X-P \beta(1-\gamma) X \sum_{j \in \mathcal{J}} \frac{\alpha_{j}^{2}}{\sum_{j^{\prime} \in \mathcal{J}} \alpha_{j^{\prime}}}-P Y+p Y
\end{aligned}
$$

where $\beta$ represents an incentive rate and $\alpha_{j} \times X \frac{\alpha_{j}}{\sum_{j^{\prime} \in \mathcal{J}} \alpha_{j^{\prime}}}$ is the shared spectrum by DAP $j$ with EUs. In the above, $\mathrm{P}$ and $\mathrm{p}$ are the prices for DAPs and EUs respectively and $\ln \left(\frac{q(W-X)}{I}\right)$ represents the gain from each NU using the spectrum $\frac{(W-X)}{I}$. In (4), $P X$ and $p Y$ are revenues of NO from sharing the spectrum $X$ and $Y$ with DAPs and EUs at prices $P$ and $p$ respectively. Term $P \beta(1-\gamma) X \sum_{j \in \mathcal{J}} \frac{\alpha_{j}^{2}}{\sum_{j^{\prime} \in \mathcal{J}^{\prime}} \alpha_{j^{\prime}}}$ is the discount amount for DAPs which is proportional to incentive rate $\beta$ and sharing rate of DAPs, where incentive rate $\beta$ diminishes by $(1-\gamma)$. On the other hand, DAPs get incentive depending on their interruption rates. Without loss of generality, we assume that the NO applies a uniform pricing for both DAPs and EUs, $P=p$. Thus, the last two terms can be dropped from the utility function (4) to obtain:

$$
\pi=I \ln \left(\frac{q(W-X)}{I}\right)+P X-P \beta(1-\gamma) X \sum_{j \in \mathcal{J}} \frac{\alpha_{j}^{2}}{\sum_{j^{\prime} \in \mathcal{J}} \alpha_{j^{\prime}}} .
$$

In DAP's side, utility can be represented as:

$$
\pi_{j}=\ln \left(X_{j} q_{j}\left(1-\alpha_{j}\right)\right)-P\left(1-\alpha_{j}\right) X_{j}+P \alpha_{j} \beta(1-\gamma) X_{j}
$$

where $X_{j}=\min \left(A_{j}, D_{j}\right)=X \frac{\alpha_{j}}{\sum_{j^{\prime} \in \mathcal{J}^{\prime}} \alpha_{j^{\prime}}}$ is the shared spectrum between NO and DAP $j, \ln \left(X_{j} q_{j}\left(1-\alpha_{j}\right)\right)$ is the gain of DAP $j$ from using the spectrum $X_{j}\left(1-\alpha_{j}\right)$ with channel efficiency $q_{j}$ for itself. DAP $j$ only needs to pay for the spectrum $\left(1-\alpha_{j}\right) X_{j}$ because it shares $\alpha_{j} X_{j}$ out of $X_{j}$ with EUs. Furthermore, it gets discount by the term $P \alpha_{j} \beta(1-\gamma) X_{j}$, where DAPs with lower $\gamma$ obtain higher incentive rates.

The utility of EU $k$ in the second market between DAPs and EUs is given by

$$
\pi_{k}=\ln \left(\frac{q(1-\gamma) Y}{K}\right)-\frac{P Y}{K}
$$

where $K$ represents the number of EUs and $q$ the channel efficiency for EUs. Every EU $k$ acquires the spectrum $\frac{Y}{K}$ at price $P$. Notice that DAPs supply spectrum for $E U$ s and get incentives from NO. The NO charges EUs according to the obtained spectrum $\frac{Y}{K}$. In (7), the shared spectrum $Y$ between APs and EUs can be calculated by

$$
Y=\sum_{j \in \mathcal{J}} \sum_{k \in \mathcal{K}} \min \left(a_{j, k}, d_{k, j}\right)=X \sum_{j \in \mathcal{J}} \alpha_{j} \frac{\alpha_{j}}{\sum_{j^{\prime} \in \mathcal{J}} \alpha_{j^{\prime}}}
$$

We differentiate utility functions of NO, DAPs and EUs with reference to $X, X_{j}$ and $Y$, respectively in order to derive supply and demand functions for a given incentive rate $\beta$ :

$$
\begin{aligned}
& \text { a) } \frac{\partial \pi}{\partial X}=0 \Rightarrow X=W-\frac{I}{P-P \beta(1-\gamma) \sum_{j \in \mathcal{J}} \frac{\alpha_{j}^{2}}{\sum_{j^{\prime} \in \mathcal{J}^{\alpha_{j^{\prime}}}}}}, \forall j \in \mathcal{J} \\
& \text { b) } \frac{\partial \pi_{j}}{\partial X_{j}}=0 \Rightarrow X_{j}=\frac{1}{P\left(1-\alpha_{j}-\alpha_{j} \beta(1-\gamma)\right)}, \forall j \\
& \text { c) } \frac{\partial \pi_{k}}{\partial Y}=\frac{1}{Y}-\frac{P}{K} \Rightarrow Y=\frac{K}{P}
\end{aligned}
$$

The market equilibrium points for this two-stage market are the points where supplies and demands are equal as follow:

$$
X=\sum_{j \in \mathcal{J}} X_{j} \wedge \sum_{j \in \mathcal{J}} \alpha_{j} X_{j}=Y
$$

where $X=\sum_{j \in \mathcal{J}} X_{j}$ and $\sum_{j \in \mathcal{J}} \alpha_{j} X_{j}=Y$ are the equilibrium points for the first and second markets. Therefore, the equilibrium price is given by

$$
\begin{aligned}
& X=\sum_{j \in \mathcal{J}} X_{j} \Rightarrow P=\frac{I}{W\left(1-\beta(1-\gamma) \sum_{j \in \mathcal{J}} \frac{\alpha_{j}^{2}}{\sum_{j^{\prime} \in \mathcal{J}^{\alpha}}{ }_{j^{\prime}}}\right)}+ \\
& \sum_{j \in \mathcal{J}} \frac{1}{W\left(1-\alpha_{j}-\alpha_{j} \beta(1-\gamma)\right)}
\end{aligned}
$$

We find an equilibrium point for the second market among DAPs and EUs as follow:

$$
\sum_{j \in \mathcal{J}} \alpha_{j} X_{j}=Y \Leftrightarrow p=\sum_{j \in \mathcal{J}} \frac{\alpha_{j}}{K\left(1-\alpha_{j}-\alpha_{j} \beta(1-\gamma)\right)}
$$

In a simpler scenario including DAPs with equal sharing rates $\alpha$, we can calculate the equilibrium price as:

$$
P=\frac{J}{W(1-\alpha-\alpha \beta(1-\gamma))}+\frac{I}{W(1-\alpha \beta(1-\gamma))}
$$




\section{B. Opportunistic Spectrum Sharing Game}

We use a Stackelberg game model (SGM) [14], [15] to solve the spectrum sharing problem with a single operator in DNA network, where NO as leader is dealing with DAPs as the first followers and EUs as the second followers. Leader and followers compete with each other for certain resources e.g. spectrum. The leader moves first and the followers move subsequently. The leader imposes price and incentive rate per unit of shared spectrum with DAPs and EUs. Then, DAPs (followers 1) update their demands $D$ and EUs (followers 2) subsequently update their demands $d$.

We use backward induction (BI) [16] [17] solution to solve this sequential game. The backward induction technique is process of "looking ahead and working backwards" to solve the subgames based on sequential rationality.

In the first step of the spectrum sharing mechanism, NO as a leader broadcasts $(P, d, \beta)$ to DAPs. Next, DAPs which are the first group of followers use $(P, d, \beta)$ to calculate their Nash equilibrium point as the solution of the non-cooperative game between DAPs. They send $(D, a)$ back to the NO. Then, the NO broadcasts $(p, a)$ to EUs. Next, EUs as the second followers cooperatively find their optimum demand $d$ and send it back to the NO. This procedure continues until all players attain the equilibrium points and have no incentive to deviate from these points.

\section{Single Operator as Leader}

We develop a double Stackelberg opportunistic spectrum sharing game (DSOSS) model including two separate Stackelberg games with the same leader and different followers. In the first game, NO is leader and DAPs are followers. The NO broadcasts $(P, d, \beta)$, then DAPs non-cooperatively calculate $(D, a)$. In the second Stackelberg game, the NO is the leader as before and EUs are followers. Note that the second game happens just after the first one until all players reach to their equilibrium points. The profit function of the NO similar to (4) is

$$
\begin{gathered}
\pi(P, p, \beta)=I U\left(q \frac{W-X}{I}\right)+\sum_{j \in \mathcal{J}} P_{j}\left(X_{j}-Y_{j}\right)+ \\
p Y-\sum_{j \in \mathcal{J}} Y_{j} P_{j} \beta\left(1-\gamma_{j}\right)
\end{gathered}
$$

where $\gamma_{j}$ represents the interruption probability of DAP $j, X_{j}$ and $Y_{j}$ denote respectively demanded and shared spectrum by $D A P j$ and $X=\sum_{j \in \mathcal{J}} X_{j}$ is the total shared spectrum by the NO. Every DAP $j$ gets $X_{j}$ from the NO and shares $Y_{j}$ with EUs. In the above, parameter $\gamma_{j}$ convinces DAP $j$ to cooperate; otherwise it looses the gain from the spectrum sharing with EUs. DAPs obtain the higher incentive rate if they avoid interrupting the ongoing transmission of EUs. In (14), the concave function $U$ reflects the gains of operator from serving its NUs by distributing bandwidth $W-X$ between them. NO handles the spectrum sharing mechanism by adjusting $(P, p, \beta)$. Thus, terms $I U\left(q \frac{W-X}{I}\right)$ in (14) is constant for a given $(X, Y)$. So, we can drop it from the objective function. Furthermore, we assume that the NO applies a uniform pricing model to DAPs and EUs, so $P=p \Rightarrow \sum_{j \in \mathcal{J}} P_{j} Y_{j}=p Y$. Now NO's profit function is given by

$$
\pi(P, \beta)=\sum_{j \in \mathcal{J}} P_{j} X_{j}-\sum_{j \in \mathcal{J}} Y_{j} P_{j} \beta\left(1-\gamma_{j}\right)
$$

Under the Stackelberg game model, NO's objective is to maximize its revenue by solving the following optimization problem:

$$
\text { Problem } \left.\left.1: \max _{P, \beta} \quad \pi \quad \text { s. t. } \quad a\right) . P \geq 0 \quad b\right) .0 \leq \beta \leq 1
$$

This is a non-convex optimization problem due to the term $\sum_{j \in \mathcal{J}} Y_{j} P_{j} \beta\left(1-\gamma_{j}\right)$ in the objective function. We can replace the non-convex term $P_{j} \beta$ with $\frac{1}{4}\left(P_{j}+\beta\right)^{2}-\frac{1}{4}\left(P_{j}-\beta\right)^{2}$ to obtain the new objective function of leader as

$$
\begin{aligned}
& \pi(P, \beta)=\sum_{j \in \mathcal{J}} P_{j} X_{j}-\frac{1}{4} \sum_{j \in \mathcal{J}} Y_{j}\left(P_{j}+\beta\right)^{2}\left(1-\gamma_{j}\right)+ \\
& \frac{1}{4} \sum_{j \in \mathcal{J}} Y_{j}\left(P_{j}-\beta\right)^{2}\left(1-\gamma_{j}\right)
\end{aligned}
$$

where the objective function is not in concave form due to the convex term $\left(P_{j}-\beta\right)^{2}$. We use the first order approximation method to linearize the term $\left(P_{j}-\beta\right)^{2}$. Therefore, the final form of the leader's objective function is given by

$$
\begin{aligned}
& \pi(P, \beta)=\sum_{j \in \mathcal{J}} P_{j} X_{j}-\frac{1}{4} \sum_{j \in \mathcal{J}} Y_{j}\left(P_{j}+\beta\right)^{2}\left(1-\gamma_{j}\right)+ \\
& \quad \frac{1}{4} \sum_{j \in \mathcal{J}} Y_{j}\left(P_{j}^{(t)}-\beta^{(t)}\right)^{2}\left(1-\gamma_{j}\right)+ \\
& \frac{1}{2} \sum_{j \in \mathcal{J}} Y_{j}\left\{\left(\left(P_{j}-P_{j}^{(t)}\right)-\left(\beta-\beta^{(t)}\right)\right)\left(P_{j}^{(t)}-\beta^{(t)}\right)\right\}\left(1-\gamma_{j}\right)
\end{aligned}
$$

where $t$ is the iteration index. Now, the optimization problem is in convex form.

\section{DAPs as First Followers}

At the APs' side, every DAP non-cooperatively optimizes its own utility function given by

$$
\begin{aligned}
& \pi_{j}\left(X_{j}, Y_{j}\right)=U_{j}\left(X_{j}, Y_{j}\right)-P_{j}\left(X_{j}-Y_{j}\right)+ \\
& Y_{j} P_{j} \beta\left(1-\gamma_{j}\right)-E_{j} Y_{j}
\end{aligned}
$$

where the concave function $U_{j}$ reflects the DAP $j$ 's benefit from using the spectrum $X_{j}-Y_{j}$ for itself and $E_{j}$ represents the energy consumption rate of DAP $j$ due to the spectrum sharing process. The second term $P_{j}\left(X_{j}-Y_{j}\right)$ in (19) is the cost that should be paid by DAP $j$ to the NO proportional to its usage. Term $Y_{j} P_{j} \beta\left(1-\gamma_{j}\right)$ is discount proportional to sharing volume $Y_{j}$ and term $Y_{j} P_{j} \gamma_{j}$ is the penalty term due to the unpredicted interruptions caused by DAP $j$ in the spectrum sharing with EUs. It is observed from utility (19) that every AP optimizes simultaneously $X_{j}$ and $Y_{j}$ for given price and incentive rate. Formaly, optimization problem of every AP can be formulated as

$$
\text { Problem2: } \left.\left.\max _{X_{j}, Y_{j}} \quad \pi_{j} \quad \text { s. t. } \quad a\right) . \sum_{j \in \mathcal{J}} X_{j} \leq W \quad b\right) . Y_{j} \leq X_{j}
$$

where constraints (a) and (b) limit the requested spectrum from NO and the shared spectrum by DAPs. Here, we propose a 
distributed solution for the DAP noncooperative optimization problem (20).

The optimization problem (20) should be centrally solved in its original form while we can decompose the original problem into subproblems to make the proposed model suitable for the large scale network. To this end, we formulate Lagrangian for the noncooperative game of DAPs with coupled constraint $\sum_{j \in \mathcal{J}} X_{j} \leq W$ among DAPs as $\ell=\sum_{j \in \mathcal{J}} \pi_{j}-\lambda_{j}\left(W-X_{j}\right)$, where $\lambda_{j}$ is the Lagrange multiplier. The subproblem which should be solved by every DAP $j$ is given as

$$
\text { Problem } \left.2-1: \max _{X_{j}, Y_{j}} \quad \pi_{j}-\lambda_{j} X_{j} \quad \text { s. t. } \quad a\right) . Y_{j} \leq X_{j}
$$

where every optimization variable is local and constraints and objective function also are in terms of local variables. The master problem can be solved iteratively and independently by sub-gradient method at each DAP by the following updates

$$
\lambda_{j}(t+1)=\lambda_{j}(t)+\iota\left(\sum_{j \in \mathcal{J}} X_{j}-W\right)
$$

where $t$ is the iteration index, $X_{j}$ represents demand of DAP $j$ and $\iota$ is the learning rate. For simplicity in notations, we use parameter $\iota$ as learning/step size throughout the paper. The proposed distributed noncooperative spectrum sharing game for the DNA network is developed as algorithm 1.

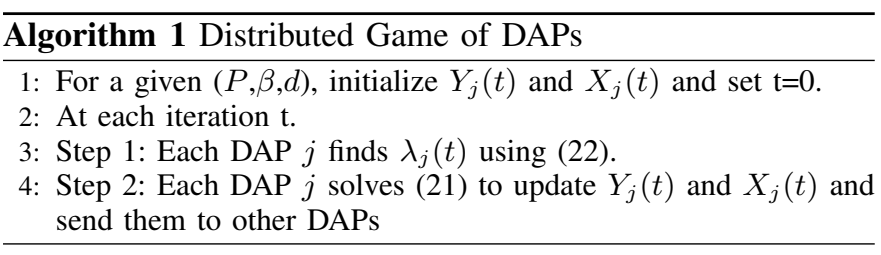

\section{E. EUs as Second Followers}

Here, we use the basic notations $D_{j}$ and $a_{j}$ for demanded and shared spectrum by DAPs when needed. To deal with the uncertainty problem in the spectrum sharing by DAPs EUs choose the most appropriate DAPs with the lowest traffic load in order to have more reliable access to the shared spectrum. So, EUs cooperatively maximize their utility for a given tuple $(a, p, X)$ by finding the optimum demand $d$ using the following utility function:

$$
\pi_{k}=\sum_{j \in \mathcal{J}} \ln \left(q_{j k}\left(1-\gamma_{j}\right) d_{k, j}\left(\frac{a_{j}-\sum_{k^{\prime} \in \mathcal{L} j(k)} d_{k^{\prime} j}}{a_{j}}\right)\right)-\sum_{j \in \mathcal{J}} d_{k j} p
$$

where $\mathcal{L}_{j}(k)$ is the cluster of EUs having coupled utility with EU $k$ when using the shared spectrum of DAP $j$, term $0 \leq \frac{a_{j}-\sum_{k^{\prime} \in \mathcal{L} j(k)} d_{k^{\prime} j}}{a_{j}} \leq 1$ is the load index of DAP $j$ and $\sum_{j \in \mathcal{J}} d_{k j} p$ is the cost paid by EU $k$ to the operator for requested spectrum $\sum_{j \in \mathcal{J}} d_{k j}$. In (23), every DAP $j$ is chosen depending on $\sum_{k^{\prime} \in \mathcal{L}_{j}(k)} d_{k^{\prime} j}$ which is the total traffic load from EUs to DAP $j$ in a cluster except EU $k$. In the above, variable $d_{k j}$ is a local variable while term $\sum_{k^{\prime} \in \mathcal{L}_{j}(k)} d_{k^{\prime} j}$ couples utility functions of EUs to each other. We assume that every EU $k$ keeps a local copy of demand of other EUs as $d^{(k)}$. So, we develop the following optimization problem for EUs:

$$
\begin{aligned}
& \text { Problem } 3: \max _{d} . \quad \pi_{k}=\sum_{j \in \mathcal{J}} \ln \left(q_{j k}\left(1-\gamma_{j}\right) d_{k j}\right)+ \\
& \ln \left(\frac{a_{j}-\sum_{k^{\prime} \in \mathcal{L}_{j}(k)} d_{k^{\prime} j}^{(k)}-d_{k j}}{a_{j}}\right)-\sum_{j \in \mathcal{J}} d_{k j} p \\
& \text { s. t. } \quad a) . \sum_{k^{\prime} \in \mathcal{L}_{j}(k)} d_{k^{\prime} j}^{(k)}+d_{k j} \leq a_{j}, \forall j \in \mathcal{J} \\
& \text { b). } d_{k j}=d_{k j}^{\left(k^{\prime}\right)}, \forall k, k^{\prime} \in \mathcal{K}
\end{aligned}
$$

where coupled constraint $d_{k j}=d_{k j}^{\left(k^{\prime}\right)}$ ensures that all copies of demands should be same. For this reason, every EU iteratively sends its demand list around. Indeed, $d_{k j}^{\left(k^{\prime}\right)}$ is a local copy of $d_{k j}$ at EU $k^{\prime}$. We apply a dual decomposition approach to relax the coupled constraint in (24). We form Lagrangian as:

$$
\ell=\sum_{k \in \mathcal{K}} \pi_{k}-\sum_{k \in \mathcal{K}} \sum_{j \in \mathcal{J}} \zeta_{k j} \sum_{k^{\prime} \in \mathcal{L}_{j}(k)}\left(d_{k j}-d_{k j}^{\left(k^{\prime}\right)}\right)
$$

where $\zeta$ is dual variable. The Lagrangian is separated into $K$ sub-problems. The local version of the optimization problem which should be run by every EU $k$ is

$$
\begin{aligned}
& \text { Problem } 3-1: \max _{d} . \pi_{k}-\sum_{j \in \mathcal{J}} \zeta_{k j} \sum_{k^{\prime} \in \mathcal{L}_{j}(k)} d_{k j} \quad \text { s. t. } \\
& \text { a). } \sum_{k^{\prime} \in \mathcal{L}_{j}(k)} d_{k^{\prime} j}^{(k)}+d_{k j} \leq a_{j}, \forall j \in \mathcal{J}
\end{aligned}
$$

The master problem can be solved iteratively and independently by sub-gradient method at each EU by

$$
\zeta_{k j}(t+1)=\zeta_{k j}(t)-\iota \sum_{k^{\prime} \in \mathcal{L}_{j}(k)}\left(d_{k j}-d_{k j}^{\left(k^{\prime}\right)}\right)
$$

Thus, every EU $k$ independently solve (26) and (27) to update its demand $d$. Then, every EU sends $d$ to other EUs.

\section{F. Subgame Perfect Equilibrium (SPE) of DSOSS}

Problems (16), (26) and (20) create two embedded Stackelberg games where the objective of these games are to find the subgame perfect equilibrium (SPE) points.

Definition 3. A strategy profile $\Xi^{*}$ is a subgame perfect equilibrium (SPE) of DSOSS if it is a Nash equilibrium (NE) of every subgame of DSOSS.

The proposed DSOSS model includes different subgames among NO, EUs and DAPs. Therefore, SPE of DSOSS is the equilibrium point where players including NO, EUs and DAPs have no incentives to leave this point.

Definition 4. Let $P, p$ and $\beta$ be a solution of (16), $D_{j}$ and $a_{j}$ be a solution of (20) for every DAP $j$ and $d$ be a solution of (26). Then the point $\left(P^{*}, p^{*}, \beta^{*}, D^{*}, a^{*}, d^{*}\right)$ is a SPE point for DSOSS if for any possible solution $(P, p, \beta, D, a, d)$, the following conditions hold:

$$
\begin{aligned}
& \text { a) } \pi\left(P^{*}, p^{*}, \beta^{*}, D^{*}, a^{*}, d^{*}\right) \geq \pi\left(P, p, \beta, D^{*}, a^{*}, d^{*}\right) \\
& \text { b) } \pi_{j}\left(P^{*}, \beta^{*}, D_{j}^{*}, a_{j}^{*}, D_{-j}^{*}, a_{-j}^{*}, d^{*}\right) \geq \\
& \quad \pi_{j}\left(P^{*}, \beta^{*}, D_{j}, a_{j}, D_{-j}^{*}, a_{-j}^{*}, d^{*}\right), \forall j \\
& \text { c) } \sum_{k \in \mathcal{K}} \pi_{k}\left(p^{*}, a^{*}, d^{*}\right) \geq \sum_{k \in \mathcal{K}} \pi_{k}\left(p^{*}, a^{*}, d\right)
\end{aligned}
$$


Inequalities $a, b$ and $c$ state that leader (NO) and followers (EUs or DAPs) choose their optimum strategy to obtain their market equilibrium points. The constraint $a$ emphasizes that NO runs its SPE strategy and it doesn't deviate from this point because it is in the optimum point. Likewise, the constraint $b$ and $c$ determine the equilibrium point of EUs and DAPs. Note that in the equilibrium points supplies and demands are equal and players will not leave these points.

In (SPE), $D_{-j}^{*}$ and $a_{-j}^{*}$ determines the optimum strategies of all DAPs except DAP $j$ in demanding and sharing phases, respectively.

A Nash equilibrium is a solution point at which no AP can achieve more utility improvement by changing its own strategy. At the NO's side, the optimum solution is obtained by solving optimization problem in (16). Finally, the optimum solution of EUs is cooperatively calculated by solving (26).

In the proposed double Stackelberg game for DNA, the SPE point can be achieved by iteratively solving problem 1, 2 and 3 . The proposed DSOSS game model for the DNA network is developed as Algorithm 2.

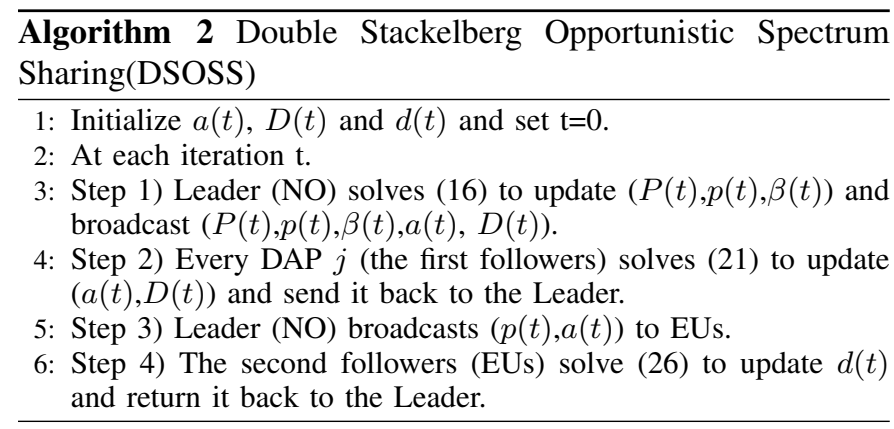

\section{Numerical Results}

In this section, the performance of the proposed models is evaluated by numerical analysis using MATLAB. We assume that in typical ultra-dense EUs are randomly placed in an area of $1 \mathrm{Km}^{2}$. Table I includes parameters used in the optimization simulations.

TABLE I

PARAMETER VALUE USED IN THE NUMERICAL ANALYSIS.

\begin{tabular}{|c|c|}
\hline Parameter & Value \\
\hline \hline$I$ & 10 \\
\hline$W$ & $10 \mathrm{~Hz}$ \\
\hline$K$ & 20 \\
\hline$\iota$ & 0.000002 \\
\hline$\gamma$ & random \\
\hline$E$ & 0.1 \\
\hline
\end{tabular}

We perform an extensive simulation campaign in order to validate the theoretical analysis of the previous sections and provide some further insights into the operation of user-centric spectrum sharing in DNA. First, we study the performance at the equilibrium points and spectrum outage probability of the proposed spectrum sharing for DNA network. Then, we consider profit and utility of NO and DAPs under the proposed Stackelberg game and optimization problems.

Fig.2 and Fig.3 show the profit (4) of NO and the utility (6) of DAP $j$ at the equilibrium point for a DNA network

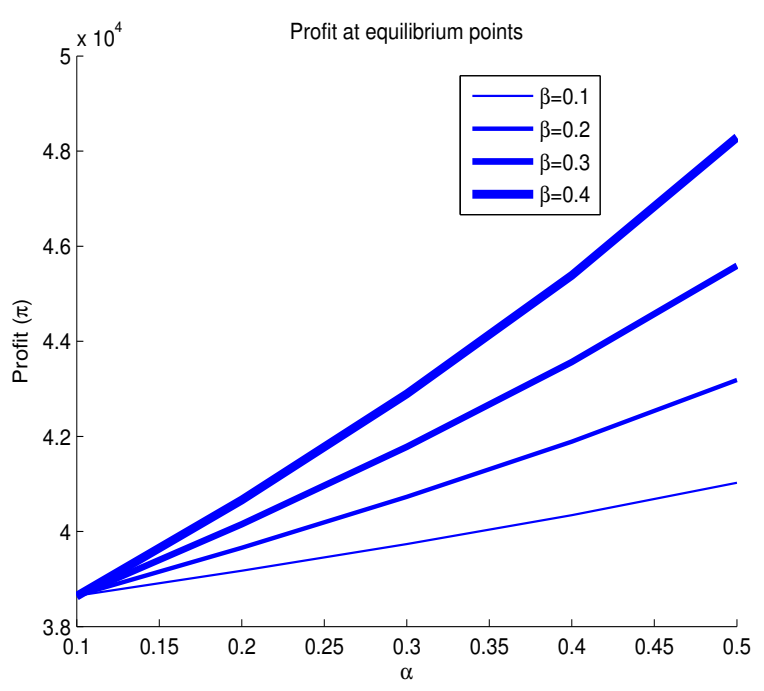

Fig. 2. Profit of $\mathrm{NO}$ at equilibrium point $(\mathrm{I}=1000, \mathrm{~J}=100, \gamma=0.1, \mathrm{~W}=10 \mathrm{~Hz})$.

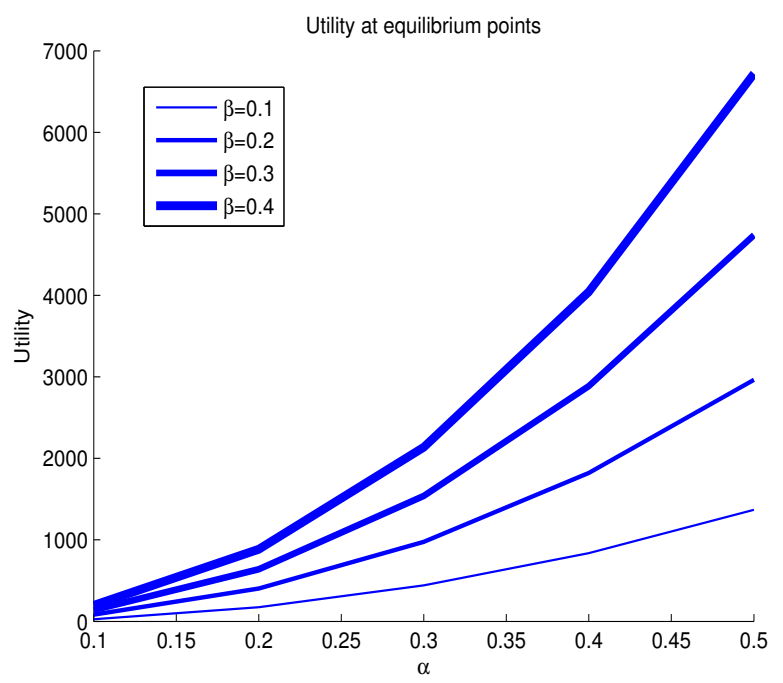

Fig. 3. Utility of DAPs at equilibrium point ( $\mathrm{I}=1000, \mathrm{~J}=100, \gamma=0.1$, $\mathrm{W}=10 \mathrm{~Hz}$ )

for different incentive and sharing rates respectively. In a homogeneous scenario when DAPs have equal arrival rates $\kappa$, the spectrum outage can be calculated by $(1-\exp (-\kappa))^{J \delta}$. In Fig. 4, the spectrum outage probability (SOP) decreases with increase of the number of DAPs and $\delta . \operatorname{In}(4)$, NO can significantly reduces SOP for EUs by introducing more DAPs to the network. Fig.5 and Fig.6 are produced by solving the proposed Stackelberg game and optimization problems. Fig.5 presents the profit of NO versus the number of AP. From the figure we can see that introducing more DAPs to the network can significantly improves the profit of NO. Furthermore, the impact of incentive rate $\beta$ can be diminished by introducing more DAPs. Fig.6 shows the average utility of DAPs for different number of DAPs in the network.

\section{CONCLUSION}

In this paper, we developed and analyzed a ubiquitous spectrum sharing mechanism where every user can share and use the spectrum. A game theoretic solution was used to model and solve the proposed spectrum sharing model. 


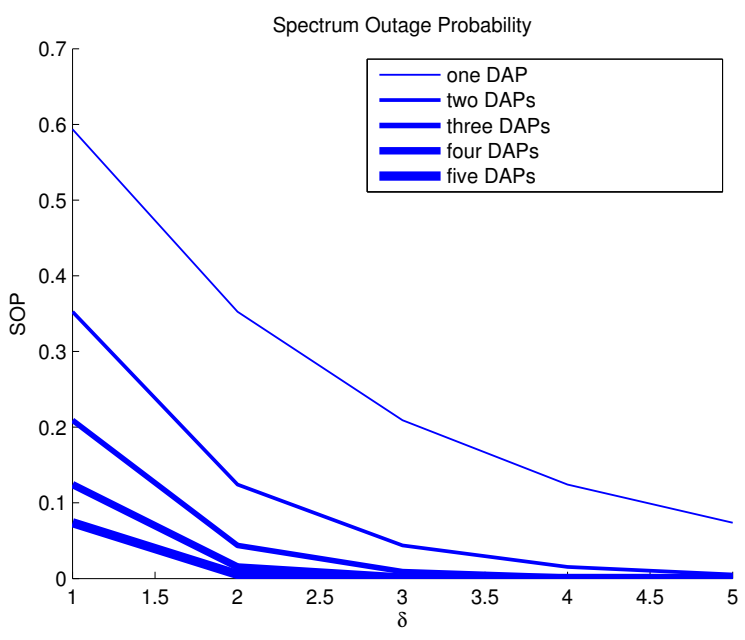

Fig. 4. The spectrum outage probability (SOP), $\kappa=0.9$.

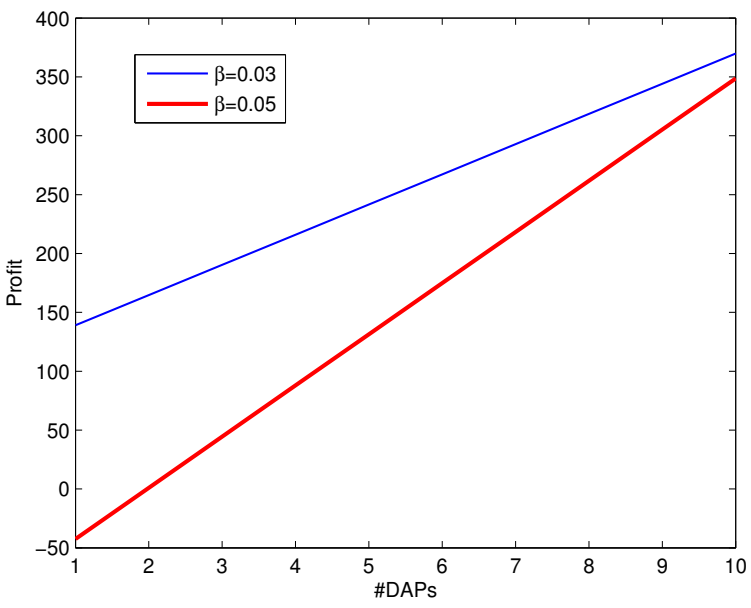

Fig. 5. Profit of NO with different $\beta$ versus the number of DAPs.

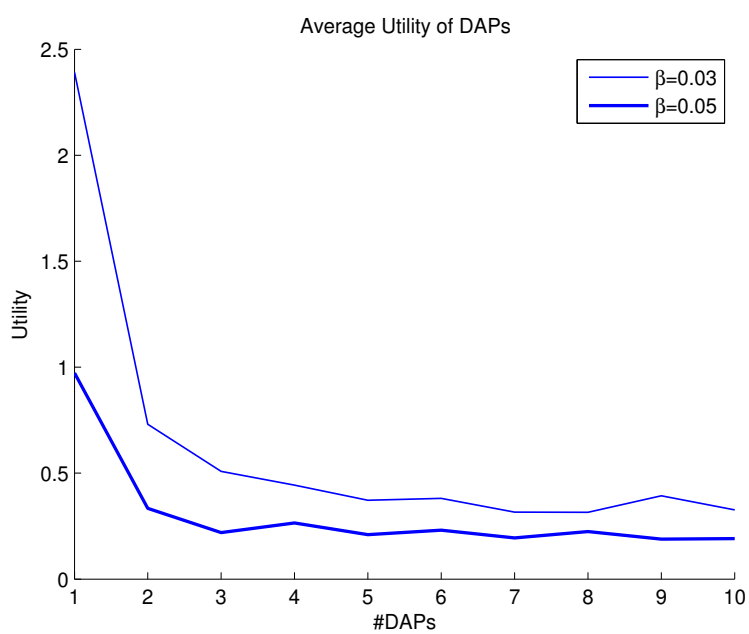

Fig. 6. Average utility of DAPs.
Furthermore, we develop a strategy to deal with uncertainty in spectrum sharing by EUs. The simulation results show a significant improvement in utilities of NOs, and DAPs if they apply a user-centric spectrum sharing model in user-provided networks.

As future work, we will consider co-channel interference as a main parameter in spectrum sharing mechanism in order to increase frequency reuse factor in ultra-dense networks.

\section{REFERENCES}

[1] R. Sofia and P. Mendes, "User-provided networks: consumer as provider," IEEE Communications Magazine, vol. 46, no. 12, pp. 8691, 2008.

[2] Y. Wang and X. Lin, "User-provided networking for qoe provisioning in mobile networks," Wireless Communications, IEEE, vol. 22, no. 4 , pp. 26-33, 2015.

[3] A. S. Shafigh, B. Lorenzo, S. Glisic, J. Perez-Romero, L. A. DaSilva, A. B. MacKenzie, and J. Roning, "A framework for dynamic network architecture and topology optimization," Networking, IEEE/ACM Transactions on, 2015.

[4] 3GPP, "Standards specifications," http://www.3gpp.org/specifications.

[5] G. Iosifidis, L. Gao, J. Huang, and L. Tassiulas, "Incentive mechanisms for user-provided networks," Communications Magazine, IEEE, vol. 52 , no. 9 , pp. 20-27, 2014

[6] V. Guerra Rolla and M. Curado, "Enabling wireless cooperation in user provided networks," in Integrated Network Management (IM), 2015 IFIP/IEEE International Symposium on. IEEE, 2015, pp. 1114-1121.

[7] L. Gao, G. Iosifidis, J. Huang, and L. Tassiulas, "Hybrid data pricing for network-assisted user-provided connectivity," in INFOCOM, 2014 Proceedings IEEE. IEEE, 2014, pp. 682-690.

[8] M. M. Khalili, L. Gao, J. Huang, and B. H. Khalaj, "Incentive design and market evolution of mobile user-provided networks," arXiv preprint arXiv:1502.06327, 2015.

[9] G. Iosifidis, L. Gao, J. Huang, and L. Tassiulas, "Enabling crowdsourced mobile internet access," in IEEE INFOCOM Proceedings. IEEE, 2014, pp. 451-459.

[10] Q. Ma, L. Gao, Y.-F. Liu, and J. Huang, "A game-theoretic analysis of user behaviors in crowdsourced wireless community networks," arXiv preprint arXiv:1503.01539, 2015

[11] Y. Zhang, Y. Gu, L. Liu, M. Pan, Z. Dawy, and Z. Han, "Incentive mechanism in crowdsourcing with moral hazard," in Wireless Communications and Networking Conference (WCNC). IEEE, 2015, pp. 20852090.

[12] S. Bayat, R. H. Louie, Z. Han, Y. Li, and B. Vucetic, "Multiple operator and multiple femtocell networks: Distributed stable matching," in Communications (ICC), 2012 IEEE International Conference on. IEEE, 2012, pp. 5140-5145.

[13] S. Bayat, R. H. Louie, Z. Han, B. Vucetic, and Y. Li, "Distributed user association and femtocell allocation in heterogeneous wireless networks," Communications, IEEE Transactions on, vol. 62, no. 8, pp. 3027-3043, 2014.

[14] D. Yang, J. R. Jiao, Y. Ji, G. Du, P. Helo, and A. Valente, "Joint optimization for coordinated configuration of product families and supply chains by a leader-follower stackelberg game," European Journal of Operational Research, vol. 246, no. 1, pp. 263-280, 2015.

[15] K. Zhu and E. Hossain, "Joint mode selection and spectrum partitioning for device-to-device communication: A dynamic stackelberg game," Wireless Communications, IEEE Transactions on, vol. 14, no. 3, pp. 1406-1420, 2015.

[16] R. J. Aumann, "Backward induction and common knowledge of rationality," Games and Economic Behavior, vol. 8, no. 1, pp. 6-19, 1995.

[17] I. Arieli and R. J. Aumann, "The logic of backward induction," Journal of Economic Theory, vol. 159, pp. 443-464, 2015. 